\title{
PROSPECTIVE, RANDOMIZED TRIAL COMPARING BLOOD AND OXYGENATED CRYSTALLOID CARDIOPLEGIA IN REOPERATIVE CORONARY ARTERY BYPASS GRAFTING
}

Jack S. Shanewise, MD

Andrzej S. Kosinski, PhD

Jorge A. Coto, MD

Ellis L. Jones, MD
Objectives: Reoperative coronary artery bypass grafting presents unique challenges for myocardial preservation. The purpose of this study was to compare oxygenated blood cardioplegia with oxygenated crystalloid cardioplegia during reoperative coronary artery bypass grafting using transesophageal echocardiography to assess regional wall motion of the left ventricle before and after cardiopulmonary bypass. Methods: Sixty-one patients undergoing reoperative coronary artery bypass grafting were prospectively randomized to receive oxygenated blood cardioplegia or oxygenated crystalloid cardioplegia delivered with a combined antegrade-retrograde technique. Transgastric short axis views of the left ventricle were made with transesophageal echocardiography during the operation before cardiopulmonary bypass and immediately after cardiopulmonary bypass. Regional wall motion was graded by a blinded observer, and before cardiopulmonary bypass scores were compared with after cardiopulmonary bypass scores. Results: No significant differences were found in the change in regional wall motion score from before cardiopulmonary bypass to after cardiopulmonary bypass between the blood and crystalloid cardioplegia groups. Conclusions: This study found blood and crystalloid cardioplegia to be equally efficacious for myocardial preservation during reoperative coronary artery bypass grafting. (J Thorac Cardiovasc Surg 1998; 115:1166-71)
$T^{\mathrm{h}}$ he number of long-term survivors of coronary artery bypass operation has steadily increased since the procedure was first performed almost 30 years ago, and as a consequence, more and more patients are undergoing reoperative coronary artery bypass grafting (rCABG). Numerous reports have documented the increased risk of rCABG in terms of mortality and complications compared with primary CABG. ${ }^{1-3}$ The presence of high-grade obstructions in the native coronary vessels and previously performed vein grafts, patent internal thoracic artery bypass grafts, and pericardial adhesions create

From the Departments of Anesthesiology and Surgery, Emory University School of Medicine, Department of Biostatistics, Rollins School of Public Health at Emory University, Atlanta, Ga.

Read at the Meeting of the Society of Cardiovascular Anesthesiologists, May 8, 1996, Salt Lake City, Utah.

Received for publication April 25, 1997; revisions requested July 21, 1997; revisions received Nov. 14, 1997; accepted for publication Nov. 14, 1997.

Address for reprints: Jack S. Shanewise, MD, Department of Anesthesiology, Emory University Hospital, 1364 Clifton Rd., Atlanta, GA 30322.

Copyright (C) 1998 by Mosby, Inc.

$0022-5223 / 98 \$ 5.00+0 \quad \mathbf{1 2 / 1 / 8 7 6 8 7}$ unique challenges for cardioplegia delivery and myocardial preservation in this population. Advances in myocardial preservation techniques such as blood-containing cardioplegia solutions and retrograde delivery through selective cannulation of the coronary sinus have been used for $\mathrm{rCABG}$, and combined antegrade-retrograde cardioplegia administration has been put forth as the preferred mode of myocardial preservation for this group. ${ }^{4}$ Nonetheless, it remains to be demonstrated whether blood or crystalloid cardioplegia solution is preferable for these often technically challenging patients. Although studies comparing these solutions have been conducted, ${ }^{5-7}$ none have addressed this issue specifically for rCABG by use of a combined delivery technique. In this study we compared oxygenated blood cardioplegia and oxygenated crystalloid cardioplegia in patients for $\mathrm{rCABG}$, both administered by means of a combined antegrade-retrograde delivery technique. We assessed the quality of myocardial preservation by evaluating and comparing regional wall motion of the left ventricle $(\mathrm{LV})$ with transesophageal echocardiography (TEE) during operation before and after cardiopulmonary bypass (CPB). 


\section{Patients and methods}

With approval of the Human Investigation Committee and informed consent, 61 consecutive patients undergoing rCABG by a single surgeon (E.L.J.) were prospectively randomized to receive oxygenated blood cardioplegia or oxygenated crystalloid cardioplegia delivered with an antegrade-retrograde technique described in detail below. After induction of anesthesia, a 5.0 MHZ TEE transducer (Hewlett-Packard, Andover, Mass.) was placed through the esophagus into the stomach. Transgastric short-axis (TGSAX) views of the LV at the midpapillary level were made during operation before CPB (preCPB) and immediately after $\mathrm{CPB}$ (offCPB) when the venous return tubing was completely clamped and images recorded on $1 / 2$-inch VHS video tape. The images were evaluated retrospectively in random order by an observer (J.S.S.) blinded to patient identification and type of cardioplegia. The TGSAX views of the LV were divided into six segments (Fig. 1). Wall motion was evaluated visually for each segment, and the regional wall motion score (RWMS) was graded as follows: $0=$ normal, $1=$ mild hypokinesis, $2=$ severe hypokinesis, 3 = akinesis, $4=$ dyskinesis. The preCPB RWMS of each segment was compared with the offCPB RWMS, and the number of segments with worse function offCPB was tabulated for each patient. Analysis 1 defined worse function as an increase in RWMS by 1 or more grades, and analysis 2 as an increase in RWMS of 2 or more grades.

\section{Cardioplegia technique}

All patients were cooled on $\mathrm{CPB}$ to $25^{\circ} \mathrm{C}$. Blood cardioplegia solution was 4 parts blood withdrawn after passing through the pump oxygenator to 1 part standard crystalloid cardioplegia solution (Plegisol) with supplemental potassium $(\mathrm{K}+)$ to achieve a final concentration of $18 \mathrm{mEq} / \mathrm{L}{ }^{8}$ The $\mathrm{PO}_{2}$ of the oxygenated blood was kept between 200 and $300 \mathrm{~mm} \mathrm{Hg}$ on CPB. Crystalloid cardioplegia solution was a standard cardioplegia solution (Plegisol) with $18 \mathrm{mEq} / \mathrm{L} \mathrm{K}+$. This was oxygenated by bubbling $100 \%$ oxygen through the solution as it circulated in the cooling coil. Studies of this technique have shown the oxygen content to be about $4 \mathrm{ml} / 100 \mathrm{ml}$ of solution. ${ }^{9}$ Both cardioplegia solutions were administered in the same manner: an initial dose of $600 \mathrm{ml}$ was delivered antegrade through a cannula in the ascending aorta on aortic crossclamp application immediately followed by $600 \mathrm{ml}$ delivered retrograde through a self-inflating coronary sinus cannula (Research Medical, Inc., Midvale, Utah). Subsequent infusions of 200 $\mathrm{ml}$ were given retrograde every 20 minutes until crossclamp removal. A total of $100 \mathrm{ml}$ of cardioplegia solution was infused into each vein graft on completion of the distal anastomosis. All distal and proximal anastomoses were performed during a single aortic crossclamp period. Distal anastomoses were performed in a sequence moving in a counterclockwise direction from the right coronary artery to the posterior descending artery to the LV branch artery to the obtuse marginal artery to the diagonal artery and finally to the left anterior descending artery.

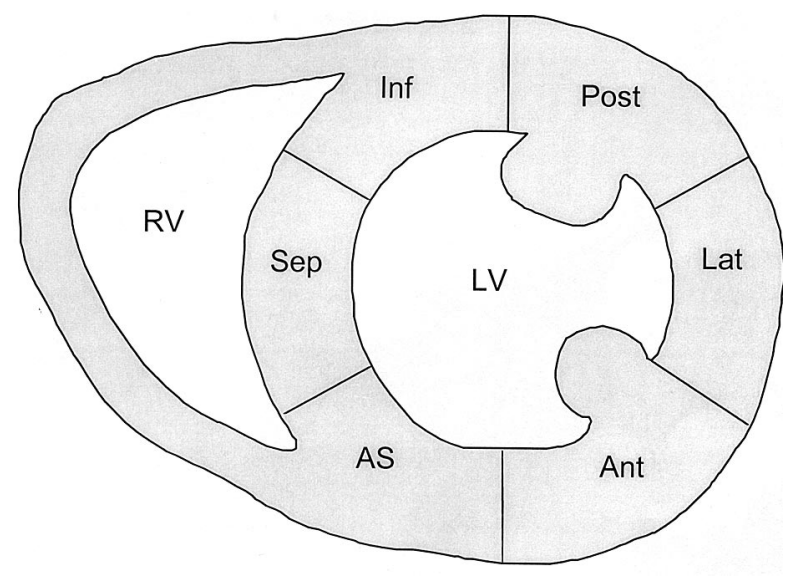

Fig. 1. Diagram of the six segment model used for the regional wall motion analysis of the TEE transgastric short axis views of the LV. $L V$, Left ventricle; $R V$, right ventricle; $A S$, anteroseptal segment; Ant, anterior segment; Lat, lateral segment; Post, posterior segment; Inf, inferior segment; Sep, septal segment.

\section{Statistical analysis}

The randomization of patients was performed according to a random permuted blocks design, with the block size varying at random. ${ }^{10}$ The randomization assignments were provided to the investigator in sealed envelopes with the patient identification number printed on the outside. For the consecutive patients, the randomization envelopes were opened once the patient was in the operating room, and only then was the indicated type of cardioplegia set up. The number of segments with increase of RWMS after $\mathrm{CPB}$ was the primary outcome variable. The comparison between the two types of cardioplegia was performed using the Fisher's exact test, ${ }^{11}$ in which the primary outcome was considered a categorical variable. For other comparisons, continuous data were displayed as a mean \pm standard deviation. Chi-square or Fisher's exact test was used to determine significance of differences for categorical variables and $t$ test or Wilcoxon two-sample test for continuous variables. ${ }^{11}$ All test were two-tailed.

\section{Results}

Thirty-one patients were assigned to blood cardioplegia and 30 patients were assigned to oxygenated crystalloid cardioplegia. No significant differences were observed between the two groups in age, sex, history of congestive heart failure, congestive heart failure class, angina class, history of myocardial infarction, severity of coronary artery disease, or preoperative ejection fraction (Table I). Significantly more patients in the oxygenated crystalloid cardioplegia group were diabetic (11 vs $4 ; p=$ 0.035). Preoperative characteristics were obtained from the hospital record, and complete information 
Table I. Preoperative characteristics

\begin{tabular}{|c|c|c|c|c|c|c|c|}
\hline \multirow[b]{2}{*}{ Characteristic } & \multicolumn{3}{|c|}{$\begin{array}{l}\text { Blood cardioplegia } \\
\quad(n=31)\end{array}$} & \multicolumn{3}{|c|}{$\begin{array}{l}\text { Oxygenated crystalloid cardioplegia } \\
\qquad(n=30)\end{array}$} & \multirow[b]{2}{*}{$p$ Value } \\
\hline & $n$ & & $(\%)$ & $n$ & & $(\%)$ & \\
\hline Age (yr) & & $n=31$ & & & $n=30$ & & 0.85 \\
\hline Mean $\pm S D$ & 64.1 & & \pm 6.9 & 63.2 & & \pm 8.8 & \\
\hline Gender & & $n=31$ & & & $n=30$ & & 0.67 \\
\hline Female & 2 & & $(6.5)$ & 3 & & $(10.0)$ & \\
\hline Male & 29 & & $(93.5)$ & 27 & & $(90.0)$ & \\
\hline History of CHF & & $n=28$ & & & $n=28$ & & 1.00 \\
\hline No & 23 & & $(82.1)$ & 24 & & $(85.7)$ & \\
\hline Yes & 5 & & $(17.9)$ & 4 & & $(14.3)$ & \\
\hline $\mathrm{CHF}$ class & & $n=27$ & & & $n=28$ & & 1.00 \\
\hline Class I & 26 & & $(96.3)$ & 26 & & $(92.9)$ & \\
\hline Class II & 0 & & $(0.0)$ & 1 & & $(3.6)$ & \\
\hline Class III & 1 & & (3.7) & 0 & & $(0.0)$ & \\
\hline Class IV & 0 & & $(0.0)$ & 1 & & (3.6) & \\
\hline Angina class & & $n=25$ & & & $n=27$ & & 0.57 \\
\hline No angina & 3 & & $(12.0)$ & 2 & & (7.4) & \\
\hline Class I & 1 & & $(4.0)$ & 0 & & $(0.0)$ & \\
\hline Class II & 3 & & $(12.0)$ & 1 & & (3.7) & \\
\hline Class III & 2 & & $(8.0)$ & 4 & & $(14.8)$ & \\
\hline Class IV & 16 & & $(64.0)$ & 20 & & $(74.1)$ & \\
\hline History of MI & & $n=24$ & & & $n=27$ & & 0.16 \\
\hline No & 10 & & $(41.7)$ & 6 & & $(23.1)$ & \\
\hline Yes & 14 & & $(58.3)$ & 20 & & (76.9) & \\
\hline No. diseased coronary arteries & & $n=31$ & & & $n=30$ & & 0.11 \\
\hline One artery & 3 & & $(9.7)$ & 1 & & $(3.3)$ & \\
\hline Two arteries & 3 & & $(9.7)$ & 9 & & $(30.0)$ & \\
\hline Three arteries & 25 & & $(80.6)$ & 20 & & $(66.7)$ & \\
\hline Ejection fraction $(\%)$ & & $n=20$ & & & $n=16$ & & 0.78 \\
\hline Mean \pm SD & 49.3 & & \pm 8.4 & 47.3 & & \pm 12.5 & \\
\hline Diabetes & & $n=28$ & & & $n=28$ & & 0.035 \\
\hline No & 24 & & $(85.7)$ & 17 & & $(60.7)$ & \\
\hline Yes & 4 & & $(14.3)$ & 11 & & $(39.3)$ & \\
\hline
\end{tabular}

$C H F$, Congestive heart failure; $M I$, myocardial infarction.

was not available for all patients. Thirty patients in the blood cardioplegia group and 26 patients in the oxygenated crystalloid cardioplegia group had TEE images suitable for analysis. No statistically significant differences were found in the number of worsening segments between the two groups with either analysis 1 , in which worse function was defined as an increase in RWMS by 1 or more grades (Fig. 2), or analysis 2 , in which worse function was defined as an increase in RWMS of 2 or more grades (Fig. 3). Twenty-three patients in the blood cardioplegia group and 24 patients in the oxygenated crystalloid cardioplegia group received infusions of inotropic and/or vasopressor drugs while being weaned from CPB $(p=0.29)$. Two patients in the blood cardioplegia group and four patients in the oxygenated crystalloid cardioplegia group had a perioperative myocardial infarction $(p=0.18)$. One patient in the blood cardioplegia group and three patients in the oxygenated crystalloid cardioplegia group died in hospital $(p=0.35)$. The patient who received blood cardioplegia died of sepsis and multiple organ system failure, and the rest died of cardiac failure. The revascularization ratio (targets/grafts performed) was similar for both groups $(1.0 \pm 0.4$ for blood cardioplegia and $1.1 \pm 0.5$ for oxygenated crystalloid cardioplegia; $p=0.46$ ), and the mean number of grafts performed was not significantly different (3.8 \pm 0.8 for blood cardioplegia and $3.4 \pm 1.2$ for oxygenated crystalloid cardioplegia; $p=0.13$ ). Mean total CPB time was longer for the oxygenated crystalloid group $(142.7 \pm 25.2 \mathrm{~min}$ for blood cardioplegia, $160.7 \pm 36.5$ min for oxygenated crystalloid cardioplegia, $p=0.04$ ). Mean aortic crossclamp time showed a trend to be longer with blood cardioplegia $(87.7 \pm 18.6 \mathrm{~min}$ for blood cardioplegia, $78.1 \pm 23.3 \mathrm{~min}$ for oxygenated crystalloid cardioplegia; $p=0.08)$. 


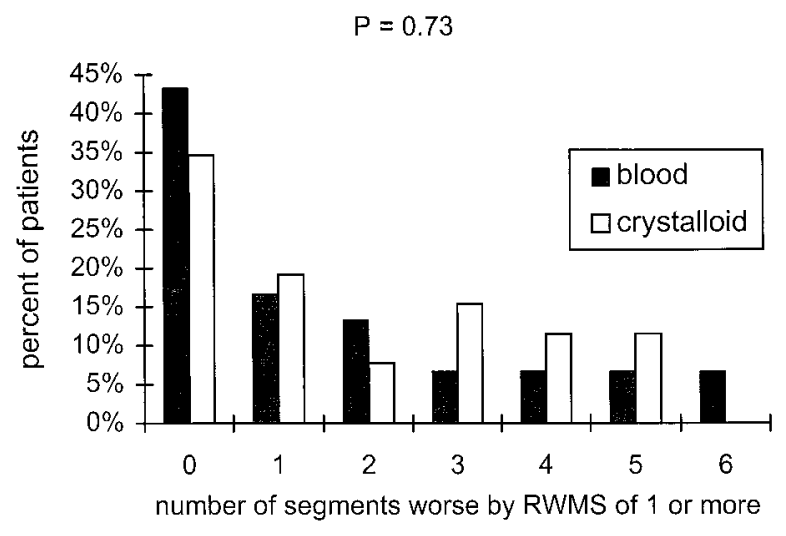

Fig. 2. Analysis 1 in which worse function of an LV segment after CPB was defined as an increase in RWMS of 1 or more. No significant difference between blood and crystalloid cardioplegia was found $(p=0.73)$. RWMS, Regional wall motion score; blood, blood cardioplegia; crystalloid, oxygenated crystalloid cardioplegia.

\section{Discussion}

This study found no difference between the blood and oxygenated crystalloid cardioplegia groups in the number of LV segments with worse RWMS after CPB. The two techniques were equally effective for myocardial preservation during $\mathrm{rCABG}$ as assessed by this means. The fact that both cardioplegia solutions were oxygenated may have tended to equalize their beneficial effects. It is also possible that a difference between the effectiveness of the techniques exists but could not be detected by a study of this size. Overall, we did not believe any clinically significant differences were found between the groups.

The use of RWMS analysis of the LV to measure the efficacy of myocardial protection in humans has not been reported per se, but it seems reasonable to assume that poorly protected portions of the LV would have a higher RWMS (less thickening) immediately after CPB than those well protected. We did not include improvement in RWMS in our analysis because we believed such changes would more likely be due to the effects of revascularization or cardiotonic drugs than cardioplegia technique, which is generally thought of as a means to preserve rather than improve myocardial function in the absence of an acute ischemic syndrome. We compared each segment with itself before and after CPB and assumed that poor myocardial preservation would result in a higher RWMS and that a technique providing better protection would result in fewer segments showing such an increase in RWMS.

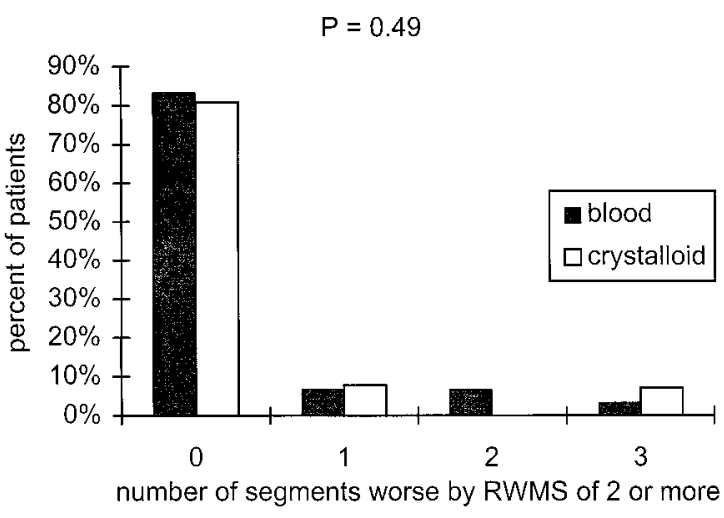

Fig. 3. Analysis 2 in which worse function of an LV segment after CPB was defined as an increase in RWMS of 2 or more. No significant difference between groups 1 and 2 was found $(p=0.49)$. RWMS, Regional wall motion score; blood, blood cardioplegia; crystalloid, oxygenated crystalloid cardioplegia.

We found no such difference between the groups in this study. Measurement of LV segmental shortening, ${ }^{12}$ as well as LV RWMS from two-dimensional echocardiographic images, ${ }^{13}$ have been used to assess myocardial protection in animal models. Another means of evaluating cardioplegia in humans is to measure the release of cardiac enzymes, such as creatine kinase or troponin, but this method does not discriminate regional from global injury, which may be of particular concern during rCABG with its combination of patent and obstructed native vessels and previously constructed bypass grafts. In addition, measuring cardiac enzymes may not detect functional disturbances not resulting in cell death. Measurement of cardiac output or LV stroke work and assessment of the need for inotropic drug or intraaortic balloon pump support after CPB have also been used to evaluate myocardial protection, but these parameters are influenced by many factors in addition to cardioplegia technique, especially as the time after CPB increases. The more remote from $\mathrm{CPB}$, the more difficult it is to attribute differences in cardiac function to cardioplegia because more and more confounding factors come into play. We evaluated change in LV RWMS from baseline before $\mathrm{CPB}$ to immediately after $\mathrm{CPB}$ to minimize the influence of factors other than myocardial protection.

This study analyzed only the TGSAX view of the $\mathrm{LV}$ and therefore did not examine the entire LV, possibly not detecting RWM changes in regions of the LV not included in this image (i.e., the apex and 
the base). It is possible that analysis of all the segments of the ventricle would have led to a different result. The TGSAX does, however, show portions of the LV supplied by all three major coronary arteries. Also, our analysis did not include the effects of blood and oxygenated crystalloid cardioplegia on the right ventricle, which is clinically important in some patients.

There are causes of changes in RWMS other than poor myocardial protection during $\mathrm{CPB}$, most notably regional perfusion abnormalities and the influence of revascularization. The number of grafts performed and the revascularization ratio of the groups were not significantly different, which argues that this influence was the same in both groups. Also, randomization should balance out any other causes of change in RWMS other than cardioplegia type, including the presence of patent but diseased bypass grafts or diffuse distal vessel disease. Warm terminal blood cardioplegic reperfusate is another technique of myocardial preservation. This was not used in this study, which was designed to compare blood cardioplegia with oxygenated crystalloid cardioplegia delivered in the same manner. The addition of a warm blood perfusate would have invalidated this comparison, and it is not routinely performed by the authors.

The oxygenated crystalloid cardioplegia group did have a higher incidence of diabetes than the blood cardioplegia group. Studies suggest that the presence of diabetes does not influence perioperative mortality or morbidity for CABG patients, ${ }^{14-16}$ and one would suspect that the higher incidence of diabetes in the oxygenated crystalloid cardioplegia group would do nothing to improve its myocardial protection and not influence the conclusion that oxygenated crystalloid cardioplegia is at least as effective as blood.

Oxygenated crystalloid cardioplegia may offer some advantages over blood cardioplegia. It provides a clearer field of vision during performance of the distal coronary anastomoses. This may be of even greater importance for $\mathrm{rCABG}$, in which tissue planes are already obscured by pericardial adhesions from the previous operation. The finding of a trend toward shorter aortic crossclamp time in the oxygenated crystalloid cardioplegia group is consistent with this opinion. On the other hand, by perfusing the coronary veins with red oxygenated blood, retrograde blood cardioplegia may facilitate their distinction from the coronary arteries, an advantage that crystalloid cardioplegia would not provide. Finally, crystalloid cardioplegia is simpler to set up and requires less equipment than blood cardioplegia, an advantage especially in this era of managed care and cost containment. Loop and colleagues ${ }^{17}$ concluded that blood cardioplegia for CABG resulted in a cost savings of more than $\$ 2000$ per case caused mainly by decreased morbidity compared with crystalloid cardioplegia, but their study was a comparison of retrospectively matched cohorts, not a prospectively randomized trial, and consisted mostly of primary CABG cases with a minority of $\mathrm{rCABG}$. It is interesting that their crystalloid cardioplegia group had a highly significantly shorter aortic crossclamp time than the blood cardioplegia group.

This study could detect no difference in the efficacy of myocardial protection provided by oxygenated blood or oxygenated crystalloid cardioplegia when administered with a combined antegraderetrograde technique to rCABG patients and would support the use of either technique as preferred by the operator.

\section{REFERENCES}

1. Lytle BW, Loop FD, Cosgrove DM, Taylor PC, Goormastic M, Peper W, et al. Fifteen hundred coronary reoperations: results and determinants of early and late survival. J Thorac Cardiovasc Surg 1987;93:847-59.

2. Saloman NW, Page US, Bigelow JC, et al. Reoperative coronary surgery: comparative analysis of 6591 patients undergoing primary bypass and 508 patients undergoing reoperative coronary artery bypass. J Thorac Cardiovasc Surg 1990;100:250-9.

3. Cameron A, Kemp HG Jr, Green GE. Reoperation for coronary artery disease: 10 years of clinical follow-up. Circulation 1988;78(Suppl I):I158-62.

4. Savage EB, Cohn LH. 'No touch' dissection, antegraderetrograde blood cardioplegia, and single aortic cross-clamp significantly reduce operative mortality of rCABG. Circulation 1994;90:II140-3.

5. Iverson LIG, Young JN, Ennix CL, et al. Myocardial protection: a comparison of cold blood and cold crystalloid cardioplegia. J Thorac Cardiovasc Surgery 1984;87:509-16.

6. Codd JE, Barner HB, Pennington DG, et al. Intraoperative myocardial protection: a comparison of blood and asanguinous cardioplegia. Ann Thorac Surg 1985;39:125-33.

7. Lajos TZ, Espersen CC, Lajos PS, et al. Comparison of cold versus warm cardioplegia. Crystalloid antegrade or retrograde blood? Circulation 1993;88:II344-9.

8. Guyton RA. Physiology and protection of the myocardium. In: Mora CT, Guyton RA, Finlayson DC, Rigatti RL, editors. Cardiopulmonary bypass: principles and techniques of extracorporeal circulation. New York: Springer-Verlag; 1995. p. 28-31.

9. Guyton RA, Dorsey LM, Craver JM, Bone DK, Jones EL, Murphy DA, et al. Improved myocardial recovery after cardioplegic arrest with an oxygenated crystalloid solution. J Thorac Cardiovasc Surg 1985;89:877-87. 
10. Pocock SJ. Clinical trials. New York: John Wiley; 1983.

11. Fisher LD, Belle GV. Biostatistics: a methodology for the health sciences. New York: John Wiley; 1993.

12. Partington MT, Acar C, Buckberg GD, Julia PL. Studies of retrograde cardioplegia II. Advantages of antegrade/retrograde cardioplegia to optimize distribution in jeopardized myocardium. J Thorac Cardiovasc Surg 1989;97:613-22.

13. Frierson JH, Penn MS, LaFont AM, et al. Effect of Buckberg cardioplegia and peripheral cardiopulmonary bypass on infarct size in the closed chest dog. J Am Coll Cardiol 1992;20:1642-9.

14. Risum O, Abdelnoor M, Svennevig JL, et al. Diabetes mellitus and morbidity and mortality risks after coronary artery bypass surgery. Scand J Thorac Cardiovasc Surg 1996;30:71-5.

15. Faglia E, Favales F, Brivio M, et al. Coronary angiography and aorto-coronary bypass surgery in type 2 diabetic patients. Diabete Metab 1995;21:420-7.

16. Clement R, Rousou JA, Engelman RM. Perioperative morbidity in diabetics requiring coronary artery bypass. Ann Thorac Surg 1988;46:321-3.

17. Loop FD, Higgins TL, Panda R, Pearce G, Estafanous FG. Myocardial protection during cardiac operations: decreased morbidity and lower cost with blood cardioplegia and coronary sinus perfusion. J Thorac Cardiovasc Surg 1992;104:60818. 